\title{
Study on Speech Compression and Decompression by using Discrete Wavelet Transform
}

\author{
Sandar 00 \\ Associate Professor, Faculty of Natural Science, University of Computer Studies, Loikaw, Myanmar
}

\begin{abstract}
How to cite this paper: Sandar Oo "Study on Speech Compression and Decompression by using Discrete Wavelet Transform" Published in International Journal of Trend in Scientific Research and Development (ijtsrd), ISSN: 24566470, Volume-3 | Issue-3, April 2019, pp.252-258, URL: http://www.ijtsrd.co $\mathrm{m} /$ papers/ijtsrd 217 27.pdf

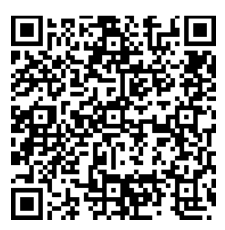

IITSRD21727

Copyright (C) 2019 by author(s) and International Journal of Trend in Scientific Research and Development Journal. This is an Open Access article distributed under the terms of the Creative Commons

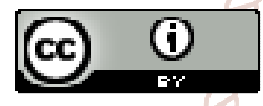
Attribution License (CC BY 4.0) (http://creativecommons.org/licenses/ by/4.0)
\end{abstract}

\section{INTRODUCTION}

Speech compression is the process of representing a voice signal for efficient transmission or storage. The compressed speech can be sent over both band limited wire and wireless channels. The aim of speech compression is to represent the samples of a speech signal in a compact form thus having the less code symbols without degrading the quality of the speech signal [1]. The compressed speech is very important in cellular and mobile communication. It is also applied in voice over internet protocol (VOIP), videoconferencing, electronic toys, archiving, digital simultaneous voice and data (DSVD), numerous computer-based gaming and multimedia applications [2].

Speech signal is compressed by converting the signal data into a new format that requires less bits to transmit. There are two basic categories of compression techniques. The first category is lossless compression. Lossless compression methods achieve completely error free decompression of the original signal. The second category is lossy compression. A lossy compression method produces inaccuracies in the decompressed signal. Lossy techniques are used when these inaccuracies are so small as to be imperceptible. The advantage of lossy technique over lossless one is that much higher compression ratios can be attained. With wavelet compression method, the imperceptible inaccuracies can be found in the decompressed signal [3].

Wavelet analysis has the benefit of varying the window size. This means that wavelets can efficiently trade time resolution for frequency resolution and vice versa. Wavelets can adapt to various time-scales and perform local analysis. Furthermore, wavelets have the ability to detect characteristics of non-stationary signals due to their finite nature that describes local features. Wavelets have been widely applied to areas such as speech and image denoising and compression [4,5]. Wavelet compression is a form of predictive compression where the amount of noise in the data set can be estimated relative to the predictive function [6].

Speech compression is the technology of converting human speech into an efficiently encoded representation that can later be decoded to produce a close approximation of the original signal. Figure 1 shows the block diagram used for compression of the speech signal and reconstruction of the signal. 


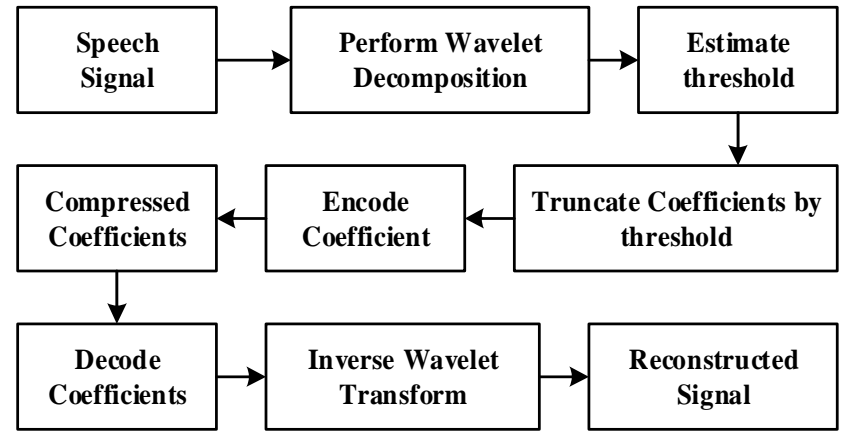

Figure 1 Block diagram used for compression and reconstruction of the speed signal.

Wavelet analysis is not a compression tool but a transformation to a domain that provides a different view of the data that is more suitable to compression than the original data itself. First the speech signal is decomposed into the wavelet transform coefficients. Then a threshold is calculated and applied to the wavelet coefficients. The small valued coefficients below a threshold are truncated to zero made an imperceptible to the signal. Signal compression is achieved by encoding the thresholder coefficients.

Many of the wavelet coefficients produced from the wavelet transform have an absolute value close to zero. These small valued coefficients are likely to attribute only small variations of the signal and contain a small percentage of the signal's total energy. These small coefficients can be discarded without a significant loss in the quality of the signal and more importantly of the interesting features. Thus, a threshold is required below which all coefficients will be discarded. The compressed signal is decoded. And then the decoded signal must be reconstructed by the inverse wavelet transform to get the original signal.

The rest of this paper is arranged as follows. In Section 2, speech compression using discrete wavelet transforms related literature to identify the key issues and summarize the experiences from various studies in different countries about the topic. In Section 3, we describe the data, the methodology and present related descriptive statistics. In Section 4, compression factors associated with fatigue driving and/or the severity of fatigue-related crashes are reported. Discussion of results is given in Section 5 .

\section{Speech Compression Using Discrete Wavelet Transform}

Speech compression using wavelets is primarily linked to the relative scarceness of the wavelet domain representation for the signal. Wavelets concentrate speech information (energy and perception) into a few neighbouring coefficients. As a result of taking the wavelet transform of the signal, many coefficients will either be zero or have negligible magnitudes. Data compression is then achieved by treating small valued coefficients as insignificant data and discarding them. The choice of wavelet, decomposition level in the discrete wavelet transform, threshold criteria for the truncation of coefficients and encoding coefficients are investigated for the process of compressing speech signal.

In the wavelet transform compression, the signal can be transformed into a wavelet domain of the signal. All values of the transform coefficients which lie below some threshold value are set to zero. Only the significant, non-zero values of the transform coefficients can be transmitted. This should be a much smaller data set than the original signal. At the receiving end, the inverse wavelet transform of the transmitted data will be performed by assigning zero values to the insignificant values which were not transmitted. This decompression produces an approximation of the original signal [3,9]. The measurement of the compression parameters is evaluated in terms of Signal to Noise Ratio (SNR), Peak Signal to Noise Ratio (PSNR), Normalized Root Mean Square Error (NRMSE) and Compression Factor (CF). The source code for speech compression will be written by using Matlab.

\section{Methodology}

\subsection{Performance \\ Measurement of Speech} Compression

A number of compression parameters can be used to evaluate the performance of the wavelet-based speech compression, in terms of both reconstructed signal quality after decoding and compression. The parameters are

$>$ Signal to Noise Ratio (SNR)

$>$ Peak Signal to Noise Ratio (PSNR)

$>$ Normalized Root Mean Square Error (NRMSE)

$>$ Retained Signal Energy (RSE)

$>$ Compression Factor $(\mathrm{CF})$

Signal to Noise Ratio: This value gives the quality of reconstructed signal.

$$
S N R=10 \log _{10}\left(\frac{\sigma_{z}^{2}}{\sigma_{e}^{2}}\right)
$$

$o_{x}^{2}$ is the mean square of the speech signal and $o_{v}^{2}$ is the mean square difference between the original and reconstructed signal.

\section{Peak Signal to Noise Ratio: $P S N R=10 \log _{10} \frac{N X^{2}}{\|x-r\|^{2}}$}

$\mathrm{N}$ is the length of the reconstructed signal, $\mathrm{X}$ is the maximum absolute square value of the signal $\mathrm{x}$ and $\|x-r\|^{2}$ is the energy of the difference between the original and reconstructed signal.

Normalized Root Mean Square Error:

$$
N R M S E=\sqrt{\frac{\left(x(n)-r(n)^{2}\right.}{\left(x(n)-\mu_{x}(n)\right)^{2}}}
$$

$x(n)$ is the speech signal, $r(n)$ is the reconstructed signal, and $\mu_{x}(n)$ is the mean of the speech signal.

Retained Signal Energy: This indicates the amount of energy retained in the compressed signal as a percentage of the energy of original signal.

$$
R S E=\frac{100 *\|x(n)\|^{2}}{\|r(n)\|^{2}}
$$

$\|x(n)\|$ is the norm of the original signal and $\|r(n)\|$ is the norm of the reconstructed signal. For one dimensional orthogonal wavelets the retained energy is equal to the $L^{2}$ norm recovery performance.

\section{Compression Factor:}

It is the ratio of the original signal to the compressed signal. The value of compression factor greater than 1 indicates compression and less than 1 indicates expansion. I referred to previous theory in my research work. 


\section{Analytical Results}

The mother wavelet chosen to compress speech signal is important as some wavelets offer better reconstruction quality and different compression ratios than others. However, there is no wavelet that gives the best results for all kinds of signals. The test signal is "Great, now we've got time to party". The test signal 'voice38kz.wav' is formed by converting the MP3 file of audio into wav file by 'wavesurfer' software. The 'voice38kz.wav' has 25913 sampled data with sampling frequency $8 \mathrm{kHz}$.

Selecting mother wavelet is related to the amount of energy a wavelet basis function can concentrate into the first level approximation coefficients. The signal energy retained in the first N/2 transform coefficients is shown in Table 1.

Table1. Signal energy retained in the first N/2 transform coefficients

\begin{tabular}{|c|c|c|c|c|c|c|}
\hline $\begin{array}{c}\text { Wav } \\
\text { elet }\end{array}$ & $\begin{array}{c}\text { Haar } \\
(\mathrm{db} 1)\end{array}$ & $\mathrm{db} 2$ & $\mathrm{db4}$ & $\mathrm{db6}$ & $\mathrm{db8}$ & $\mathrm{db} 10$ \\
\hline \multirow{2}{*}{ Ea } & $\begin{array}{c}96.32 \\
82\end{array}$ & $\begin{array}{c}98.79 \\
37\end{array}$ & $\begin{array}{c}99.40 \\
76\end{array}$ & $\begin{array}{c}99.51 \\
22\end{array}$ & $\begin{array}{c}99.57 \\
28\end{array}$ & $\begin{array}{c}99.59 \\
49\end{array}$ \\
\hline \multirow{2}{*}{$\mathrm{Ed}$} & $\begin{array}{c}3.671 \\
8\end{array}$ & $\begin{array}{c}1.206 \\
3\end{array}$ & $\begin{array}{c}0.592 \\
4\end{array}$ & $\begin{array}{c}0.487 \\
8\end{array}$ & $\begin{array}{c}0.427 \\
2\end{array}$ & $\begin{array}{c}0.405 \\
1\end{array}$ \\
\hline
\end{tabular}

This energy is equivalent to the energy stored in the first level approximation coefficients. The higher the amount of energy in the first level approximation, the better is the wavelet for compression of the signal. The Haar and Daubechies (db2, db4, db6, db8, db10) wavelets concentrate more than $96 \%$ of the signal energy. Db10 wavelet concentrates $99.5949 \%$ of energy into the first level approximation coefficients. Wavelets with many vanishing moments should be utilized for better reconstruction quality as less distortion and more signal energy concentration are introduced in the approximation coefficients. Wavelets with many vanishing moments are described with many coefficients in the scaling and wavelet functions. Thus, the computation of the wavelet transforms, the complexity of the algorithm and the output file size are increased. Figure 3 shows the flow chart of the program for compression of the speech signal.

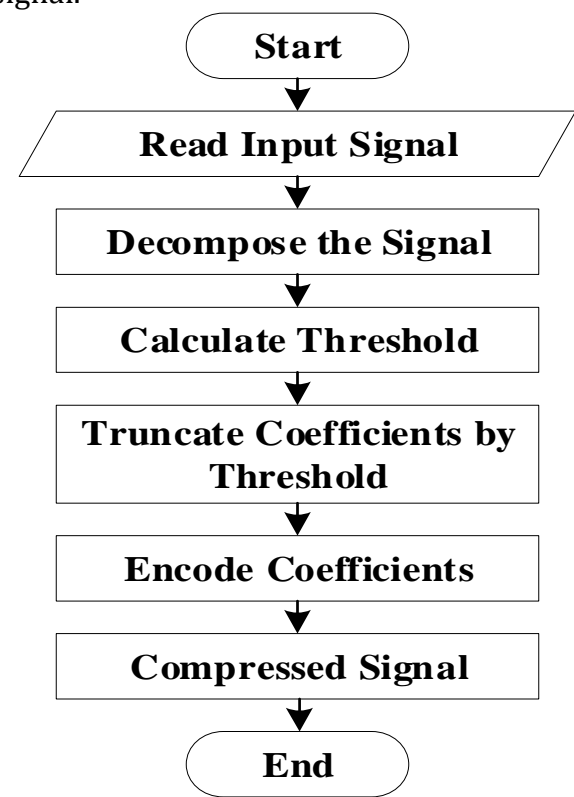

Figure3. Flow chart of the program for compression of the speech signal
In this work, the six wavelets are chosen and compared for speech compression. Choosing a decomposition level for the discrete wavelet transform usually depends on the type of signal being analyzed. For processing speech signal no advantage is gained in going beyond level 5[8]. After calculating the wavelet transform of the speech signal, compression involves truncating wavelet coefficients below a threshold. For the truncation of small valued transform coefficients, level dependent thresholding is used. Haar and Daubechies (db2, db4, db6, db8, db10) wavelets are used and compared against each other to measure the compression parameters for the speech signal. The signal is decomposed at scale 5 and level dependent threshold is applied. Figure 4 shows the flow chart of the program for decompression of the signal.

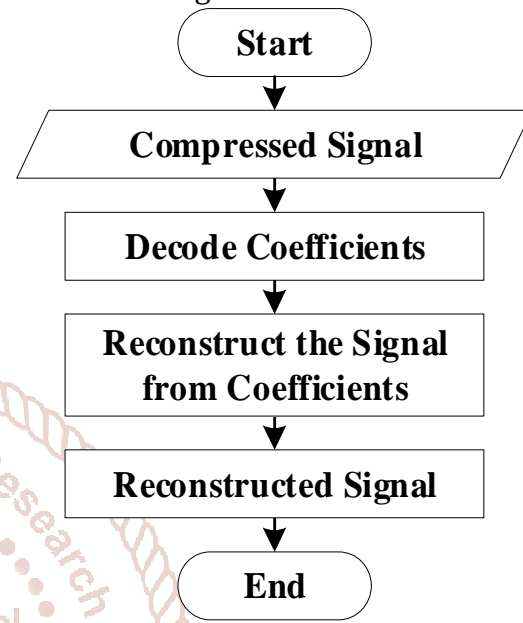

Figure 4. Flow chart of the program for decompression of the speech signal

The flow chart of the program for calculation of compression parameters is shown in Figure 5.

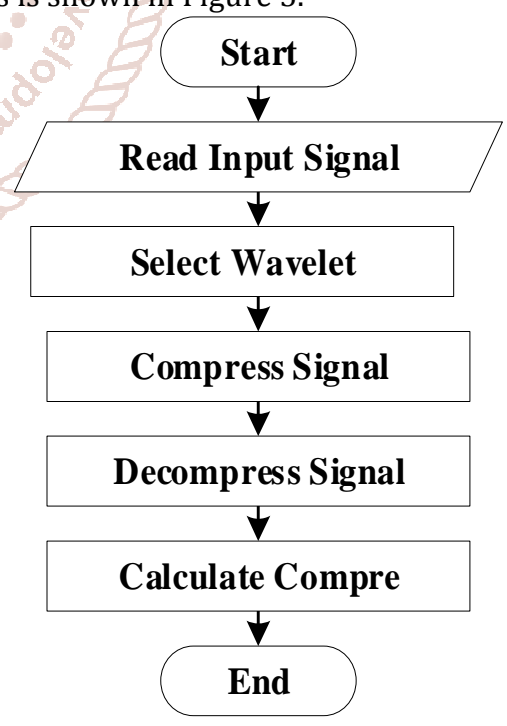

Figure5. Flow chart of the program to calculate the compression parameters

The results of the compression parameters are shown in Table 2 to Table 6 . The quality of the reconstruction signal is compared with the original signal by using SNR, PSNR and NRMSE. The higher SNR, PSNR and the lower NRMSE values give the better quality of reconstructed signal. $\mathrm{Db} 10$ wavelet gives the higher value of SNR, PSNR and the lower value of NRMSE than other wavelets in decomposition level 1. 
International Journal of Trend in Scientific Research and Development (IJTSRD) @ www.ijtsrd.com eISSN: 2456-6470

Table2. Measurement of compression parameters with different wavelets at level 1 decomposition

\begin{tabular}{|c|c|c|c|c|c|c|}
\hline Wavelet & RSE (\%) & Zeros (\%) & SNR & PSNR & NRMSE & CF \\
\hline Haar & 99.9627 & 33.4337 & 34.2806 & 47.0935 & 0.0193 & 1.3229 \\
\hline db2 & 99.9916 & 33.4118 & 40.7657 & 53.5786 & 0.0092 & 1.3272 \\
\hline db4 & 99.9960 & 33.3951 & 43.9631 & 56.7760 & 0.0063 & 1.3331 \\
\hline db6 & 99.9971 & 33.3783 & 45.3533 & 58.1661 & 0.0054 & 1.3409 \\
\hline db8 & 99.9974 & 33.3655 & 45.8720 & 58.6848 & 0.0051 & 1.3412 \\
\hline db10 & 99.9977 & 33.3488 & 46.4274 & 59.2403 & 0.0048 & 1.3461 \\
\hline
\end{tabular}

In decomposition level from 2 to 5 , the SNR, PSNR values of db10 wavelet are not obviously higher than db8 wavelet. But db 10 wavelet is better than db8. Thus, db10 wavelet gives the best result among other wavelets. RSE is the amount of energy retained in the compressed signal as a percentage of the energy of original signal. RSE is over $95 \%$ for decomposition level up to 3. The value of RSE is lesser in decomposing at scale 4 and the least value at scale 5 . The compression factor and the \% of zero coefficients are increased with increase in decomposition level. Figure 6 shows the original signal.

Table3. Measurement of compression parameters with different wavelets at level 2 decomposition

\begin{tabular}{|c|c|c|c|c|c|c|}
\hline Wavelet & RSE (\%) & Zeros (\%) & SNR & PSNR & NRMSE & CF \\
\hline Haar & 99.0978 & 61.8985 & 20.4471 & 33.2600 & 0.0950 & 2.0556 \\
\hline $\mathrm{db} 2$ & 99.7497 & 61.8759 & 26.0150 & 38.8279 & 0.0500 & 2.0970 \\
\hline $\mathrm{db} 4$ & 99.8829 & 61.8453 & 29.3154 & 42.1283 & 0.0342 & 2.1634 \\
\hline $\mathrm{db} 6$ & 99.9072 & 61.8185 & 30.3266 & 43.1394 & 0.0305 & 2.1732 \\
\hline $\mathrm{db} 8$ & 99.9253 & 61.7917 & 31.2682 & 44.0811 & 0.0273 & 2.1772 \\
\hline $\mathrm{db} 10$ & 99.9315 & 61.7765 & 31.6415 & 44.4544 & 0.0262 & 2.1929 \\
\hline
\end{tabular}

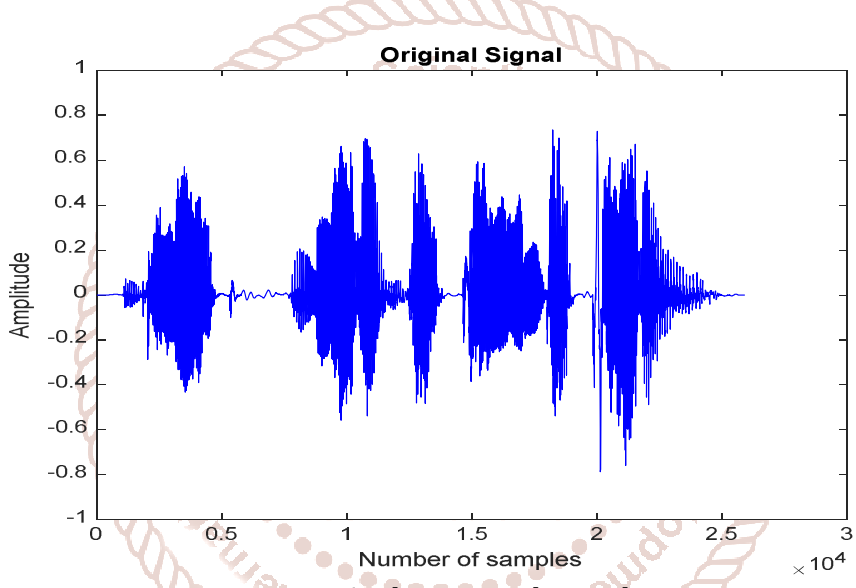

Figure6. The original signal

The comparison between the original signal and reconstructed signal using db10 wavelet is shown in Figure 7 for decomposition level 3, in Figure 8 for level 4 and in Figure 9 for level 5 respectively.

Table4. Measurement of compression parameters with different wavelets at level 3 decomposition

\begin{tabular}{|c|c|c|c|c|c|c|}
\hline Wavelet & RSE (\%) & Zeros (\%) & SNR & PSNR & NRMSE & CF \\
\hline Haar & 95.4534 & 79.3718 & 13.4231 & 26.2360 & 0.2132 & 3.5037 \\
\hline db2 & 98.0735 & 79.3519 & 17.1524 & 29.9652 & 0.1388 & 3.6080 \\
\hline db4 & 98.9267 & 79.3198 & 19.6928 & 32.5056 & 0.1036 & 3.7934 \\
\hline db6 & 99.0711 & 79.3054 & 20.3203 & 33.1332 & 0.0964 & 3.8395 \\
\hline db8 & 99.2110 & 79.2888 & 21.0290 & 33.8419 & 0.0888 & 3.8659 \\
\hline db10 & 99.2258 & 79.2783 & 21.1116 & 33.9244 & 0.0880 & 3.8670 \\
\hline
\end{tabular}

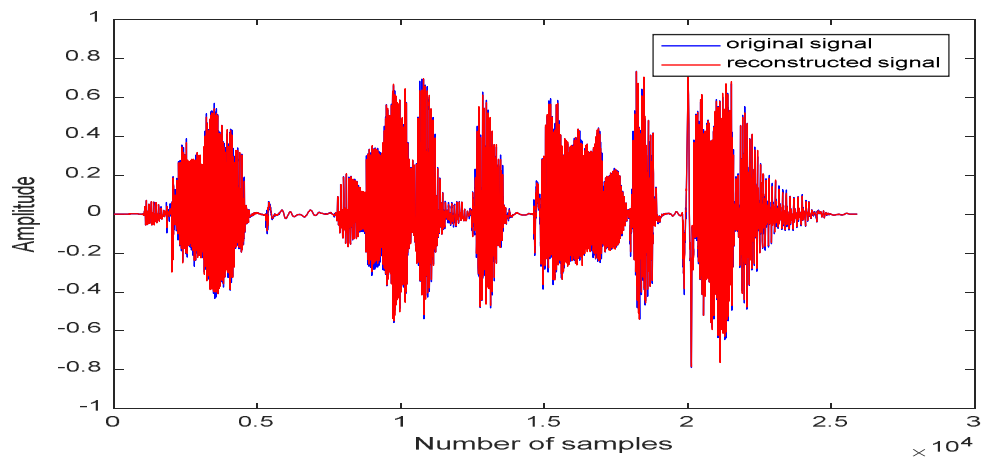

Figure7. The comparison between the original signal and reconstructed signal at decomposition level 3 
International Journal of Trend in Scientific Research and Development (IJTSRD) @ www.ijtsrd.com eISSN: 2456-6470

Table5. Measurement of compression parameters with different wavelets at level 4 decomposition

\begin{tabular}{|c|c|c|c|c|c|c|}
\hline Wavelet & RSE (\%) & Zeros (\%) & SNR & PSNR & NRMSE & CF \\
\hline Haar & 85.3250 & 89.1187 & 8.3342 & 21.1471 & 0.3831 & 6.2957 \\
\hline $\mathrm{db} 2$ & 89.4580 & 89.0985 & 9.8080 & 22.6209 & 0.3233 & 6.4557 \\
\hline $\mathrm{db} 4$ & 91.3590 & 89.0825 & 10.6344 & 23.4472 & 0.2940 & 6.7412 \\
\hline $\mathrm{db} 6$ & 91.7306 & 89.0576 & 10.8252 & 23.6381 & 0.2876 & 6.8336 \\
\hline $\mathrm{db} 8$ & 91.9858 & 89.0455 & 10.9614 & 23.7743 & 0.2831 & 6.7210 \\
\hline $\mathrm{db} 10$ & 92.1907 & 89.0137 & 11.0739 & 23.8867 & 0.2795 & 6.7888 \\
\hline
\end{tabular}

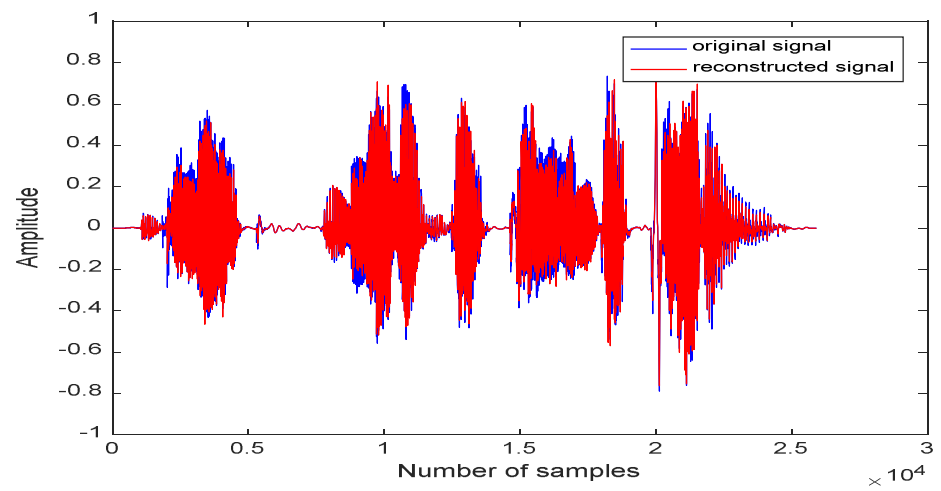

Figure8. The comparison between the original signal and reconstructed signal at decomposition level 4

Table6. Measurement of compression parameters with different wavelets at level 5 decomposition

\begin{tabular}{|c|c|c|c|c|c|c|}
\hline Wavelet & RSE (\%) & Zeros (\%) & SNR & PSNR & NRMSE & CF \\
\hline Haar & 65.7828 & 94.3471 & 4.6576 & 17.4704 & 0.5850 & 11.5631 \\
\hline $\mathrm{db} 2$ & 70.7420 & 94.3259 & 5.3375 & 18.1504 & 0.5409 & 11.5528 \\
\hline $\mathrm{db} 4$ & 72.6046 & 94.3036 & 5.6232 & 18.4361 & 0.5234 & 11.6936 \\
\hline $\mathrm{db} 6$ & 73.5452 & 94.2692 & 5.7750 & 18.5878 & 0.5143 & 11.8541 \\
\hline $\mathrm{db} 8$ & 74.3177 & 94.2392 & 5.8037 & 18.6165 & 0.5068 & 11.6883 \\
\hline $\mathrm{db} 10$ & 74.2488 & 94.2167 & 5.8920 & 18.7049 & 0.5075 & 11.5169 \\
\hline
\end{tabular}

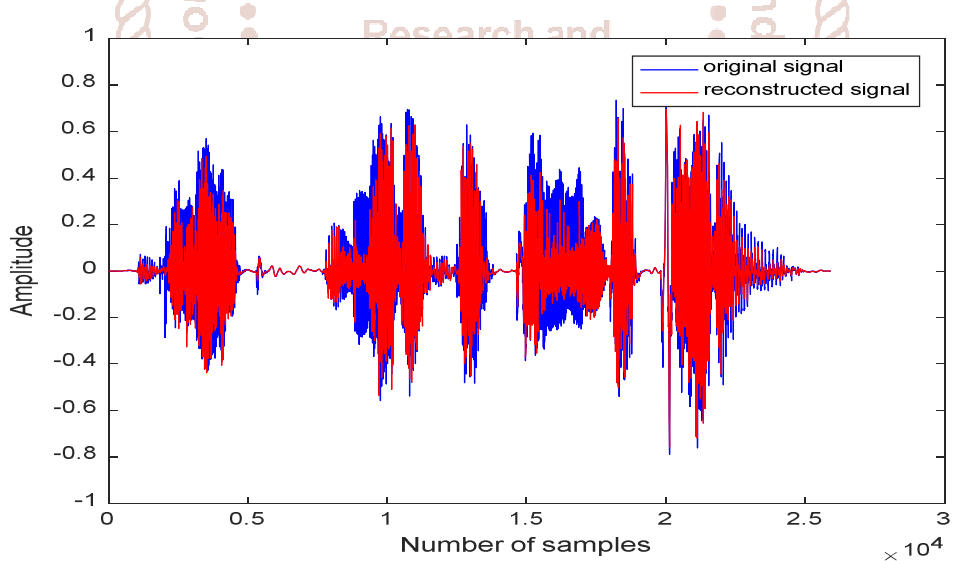

Figure9. The comparison between the original signal and reconstructed signal at decomposition level 5

\section{Results and Discussion}

The reconstructed signal is written to an audio file by using 'audiowrite' function in Matlab. Listening test is carried out on each level of the reconstructed signal in audio file. The quality of reconstructed signal is very close to the original signal in the decomposition level 1 and 2 . The quality of the signal is nearly close to the original signal in level 3 . The quality of the reconstructed signal is bad at decomposition level 4 and 5. From the overall results the level 3 decomposition is suitable for this signal. At higher levels the approximation data is not as significant and hence does a poor job in approximating the input signal. The number of samples in the compressed signal with different wavelets are shown in Table 7.

Table 7. The number of samples in the compressed signal by six wavelets for decomposition level up to 5

\begin{tabular}{|c|c|c|c|c|c|}
\hline Wavelet & Level 1 & Level 2 & Level 3 & Level 4 & Level 5 \\
\hline Haar & 19588 & 12606 & 7396 & 4116 & 2241 \\
\hline $\mathrm{db} 2$ & 19525 & 12357 & 7182 & 4014 & 2243 \\
\hline $\mathrm{db} 4$ & 19438 & 11978 & 6831 & 3844 & 2216 \\
\hline $\mathrm{db} 6$ & 19325 & 11924 & 6749 & 3792 & 2186 \\
\hline $\mathrm{db} 8$ & 19321 & 11902 & 6696 & 3799 & 2217 \\
\hline $\mathrm{db} 10$ & 19250 & 11817 & 6701 & 3817 & 2250 \\
\hline
\end{tabular}


Figure 10 shows the results of compression parameters using db10 wavelet with different decomposition levels.

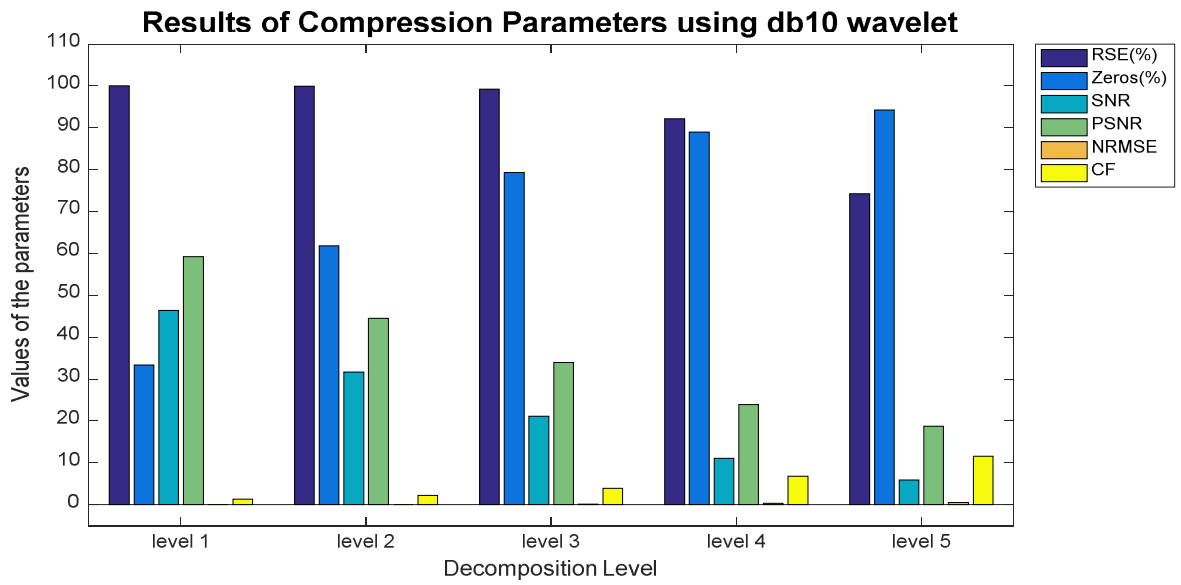

Figure10. The results of compression parameters using db10 wavelet with different decomposition levels

The comparison result of the original signal and compressed signal is shown in Figure 11.

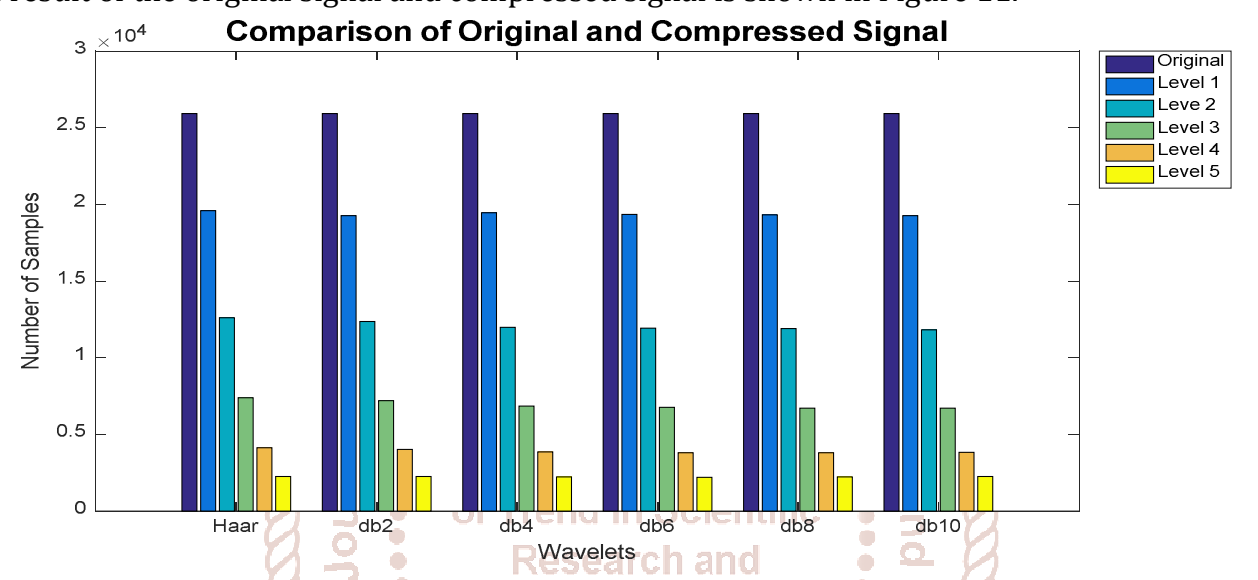

Figure11. The comparison of the original and compressed signal at decomposition level up to 5

The signal to noise ratio variation relative to compression factors using db10 wavelet is shown in Figure 12 . The source code for the calculation of compression parameters is displayed in Matlab.

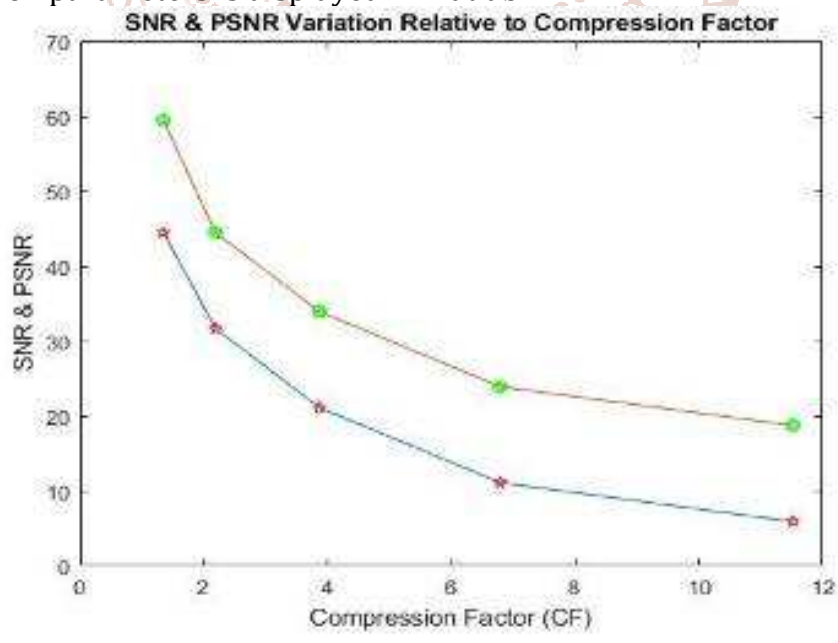

Figure 12.SNR and PSNR variation relative to compression factors using db10 wavelet

\section{Conclusion}

Speech compression is a solution to the problem of large amount of storage and bandlimited transmission. The discrete wavelet transform performs well in the compression of speech signal. The performance measurement results are obtained by using the Haar and Daubechies wavelets. The compressed signal can be reconstructed back to its original form with full audibility. A good reconstructed signal is the one with low MSE and high
PSNR and SNR. This means that the signal has low error and high signal fidelity. Db10 wavelet has the high SNR, PSNR values and the low NRMSE as compared with other wavelets. SNR, PSNR, NRMSE, CF, RSE and \% of zero coefficients are measured to evaluate the performance of the speech compression. Decomposition level at scale 3 is suitable for this signal. The measurement results are obtained by writing the source code in Matlab.The decomposition level for different types of speech will be chosen using wavelets. 


\section{References}

[1] Jagtap S.K., Mulye M S., and Uplane M.D., (2015), “ Speech Coding Techniques", the 4th International Conference on Advances in Computing, Communication and Control, London: Elsevier Inc./S\&T Books and Cell Press

[2] Sun L., Mkwawa I.H., Jammeh E., and Ifeachor E., (2013), "Guide to Voice and Video over IP", Computer Communications and Networks, UK: Springer-Verlag London Ltd.

[3] WALKER J.S., (2008), "A Primer on WAVELETS and their Scientific Applications", 2nd Edi, USA: Chapman \& Hall/CRC Press

[4] Kyriakopoulos, K.G., and Parish D.J., (2010), “ Applying Wavelets for the Controlled Compression of Communication Network Measurements", Loughborough University's Institutional Repository, pp. 507-520
[5] Michel M., Yves M., Georges O., and Poggi J.M.,(2010), "Wavelet Toolbox User's Guide", the Math Works, Inc.

[6] Petkar H, (2017), "Speech Compressing using Wavelet Transform", IOSR Journal of Computer Engineering, Vol. 19, Issue 3, May, pp.33-41

[7] Rajput A,Q., "Wavelet Based Speech Compression for VOIP Applications", Institute of Information Technology, Mehran University of Engineering \& Technology, Jamshoro. Sindh Pakistan

[8] Rao N., and Homer J., (2001), " Speech Compression using Wavelets", Information Technology and Electrical Engineering, the University of Queensland, October

[9] Winkler J.R., "Orthogonal Wavelets via Filter Banks: Theory and Applications", the University of Sheffield, Department of Computer Science, United Kingdom, pp.122

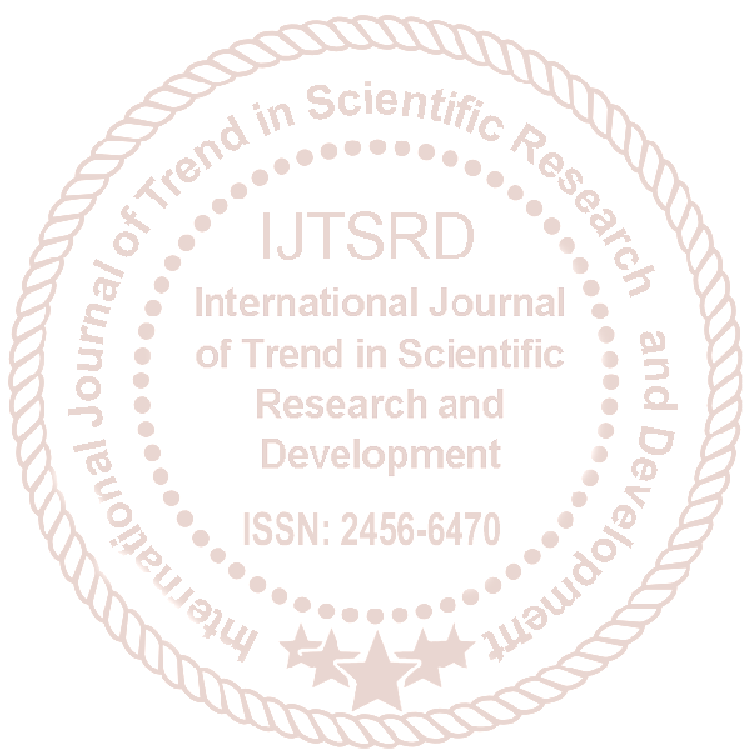

